\title{
NOTES ON A PAPER OF MESS
}

\author{
LARS ANDERSSON, THIERRY BARBOT, RICCARDO BENEDETTI, FRANCESCO \\ BONSANTE, WILLIAM M. GOLDMAN, FRANÇOIS LABOURIE, KEVIN P. SCANNELL, \\ AND JEAN-MARC SCHLENKER
}

\section{N.1. INTRODUCTION}

In his 1990 paper "Lorentz Spacetimes of Constant Curvature" 82, Geoff Mess offered what was, at the time, a completely new approach to the study of spacetimes in $2+1$-dimensions, primarily by employing tools and techniques from lowdimensional geometry and topology. Among the many interesting results in the paper is a complete geometric parameterization of the moduli space of flat $2+1$ spacetimes with closed Cauchy surfaces. By "geometric" we mean that the parameterization is defined in terms of certain objects that arise in the Cauchy horizons of such spacetimes (specifically $\mathbb{R}$-trees, or, dually, measured laminations - structures introduced by Thurston in his groundbreaking work on hyperbolic 3-manifolds in the 1970's). This allows one to do much more than merely count degrees of freedom in the moduli space. For example, certain previously inaccessible problems can be resolved quite easily in this language, such as results on the inextendability of domains of dependence past the Cauchy horizon (Cosmic Censorship); see $\$ \mathbf{N . 5 . 3}$ below.

The paper was written around the same time that Witten and others began analyzing $2+1$-gravity as a kind of toy model for quantization, and it attracted a lot of attention (and many citations) from the physics community. It has been similarly influential within mathematics, with applications to 3-dimensional affine geometry, the deformation theory of hyperbolic manifolds, etc.

Unfortunately, the paper was never published. In addition, it has never been particularly easy to find in preprint form since there was no electronic version available. For this reason, and because of the profound impact the paper has had, we decided that it would be a useful service to the community to have the paper in print, even in its somewhat unpolished state.

The version of the paper that follows is nearly identical to the original; we merely corrected typographical errors and occasional notational mistakes, and also updated the references in the bibliography. The numbering of definitions, propositions, and theorems is unchanged, so that earlier citations to the preprint can easily be traced in this version.

These "Notes" are intended as a roadmap to Mess' paper, which can be difficult to follow at times. We offer some simplifications to the original arguments, an

Date: February 11, 2013.

1991 Mathematics Subject Classification. 83C80 (83C57), 57S25.

Key words and phrases. flat spacetimes, Minkowski space, anti-de Sitter, de Sitter, causality, globally hyperbolic.

The first author was supported in part by the NSF, contract no. DMS 0407732.

The second author was supported by CNRS, ACI "Structures géométriques et Trous Noirs". 
occasional correction, and most importantly a guide to the literature on this subject that has appeared in the intervening 16 years.

N.2 - $\$ \mathbf{N} .8$ of the present paper correspond to the sections of Mess' paper with the same numbers, for easy cross-referencing while reading the latter. These notes also contain two additional sections describing some recent generalizations and improvements upon Mess' results. In $\$ \overline{N .9}$ we discuss an important unifying notion (the canonical Wick rotation) due to Benedetti and Bonsante. The final section (\$N.10) considers slicings of spacetimes by constant mean curvature surfaces, a problem raised by Mess but not addressed directly in his paper.

Definitions, propositions, and theorems in these Notes are numbered with a prefixed "N" (Lemma N.3.1, Corollary N.3.2, etc.), to avoid confusion with the numbering in Mess' paper.

The authors would like to thank Dick Canary, David Garfinkle, Steve Harris, Misha Kapovich, Steve Kerckhoff, Cyril Lecuire, John Ratcliffe, and Ser Tan for helpful correspondence. Thanks to Taejung Kim for helping typeset Mess' manuscript, and to the referee for a number of useful comments.

\section{N.2. FuCHSIAN HOLONOMY}

The main result of this section is Proposition 1; this says that the linear part of the holonomy of a closed spacelike surface with $g>1$ in a flat $2+1$ spacetime is Fuchsian. The proof is based on the Milnor-Wood inequality [97] and Goldman's theorem [59] and there is a terse exposition of these results (the proof of Goldman's theorem being essentially Matsumoto's [80]). These are best read from the original sources.

A second proof of discreteness of the linear holonomy is sketched at the end of $\S 8$; see [17] for details.

Proposition 2 is the simple observation that any Fuchsian group gives a flat spacetime by quotienting the interior of the future (or past) light cone.

\section{N.3. ReAlizATION OF HOLONOMY hOMOMORPHISMS}

The main result is Proposition 3, which is an application of the holonomy theorem to say that a small affine deformation of a given Fuchsian linear representation of a closed surface group is realized by a future complete flat spacetime; rescaling then realizes any affine deformation in this way.

The fact that the surface is closed is critical here, since there are examples of affine deformations of Fuchsian groups with parabolics that do not leave invariant a future complete regular convex domain [17, as well as the "crooked plane" examples of Goldman and Drumm [46] which, in contrast with the examples constructed by Mess, actually act discontinuously on all of Minkowski space.

Also critical to the later development is Lemma 1, which shows that a spacelike immersion of a surface into Minkowski space such that the induced metric is complete must actually be an embedding. In later sections this is applied to the developing map of the universal cover of a Cauchy surface.

We provide here a particularly nice geometric argument generalizing Lemma 1; this argument was known previously (at least in [61]).

This argument applies in any Lorentzian manifold $M$ admitting a complete Killing vector field $X$ which is everywhere timelike (in particular, non-singular), 
and such that, if $\Phi^{t}$ denotes the flow generated by $X$, every orbit of $\Phi^{t}$ is wandering, i.e., at any point $x$ of $M$ there is a small transversal $D$ to $\Phi^{t}$ containing $x$ and such that $D$ intersects every $\Phi^{t}$-orbit at most once. Observe that if $M$ is strongly causal (see $\$ \mathrm{N.5.1}$ ), then this hypothesis is automatically satisfied.

This hypothesis implies that the orbit space $Q^{\Phi}$ is a manifold, though possibly non-Hausdorff: non-Hausdorffness might appear from ancestral pairs; see [62. It can be shown that under reasonable hypotheses, valid in Minkowski and anti-de Sitter space, $Q^{\Phi}$ is Hausdorff: for example, if $M$ admits a time function and is timelike or null geodesically complete (Theorem 2 in [55]).

Let $\pi: M \rightarrow Q^{\Phi}$ be the projection map. For any tangent vector $\bar{v}$ to $Q^{\Phi}$ at a point $\theta$, define $g_{0}(\bar{v})$ to be the norm in $M$ of any vector $v$ orthogonal to $X(x)$ such that $x \in \theta$ and $d \pi(v)=\bar{v}$. Since $X$ is Killing, this norm does not depend on the choice of the point $x$ in the orbit $\theta$. It defines a Riemannian metric $g_{0}$ on $Q^{\Phi}$. Observe also that $\pi$ is a locally trivial fibration. The proof of the following lemma is straightforward:

Lemma N.3.1. Let $S$ be a Riemannian manifold, and let $f: S \rightarrow M$ be a codimension 1 isometric immersion. Then, the composition $\pi \circ f$ is distance increasing.

Corollary N.3.2. If the Riemannian metric on $S$ is complete, then $\pi \circ f$ is a covering map by a standard argument [69].

Corollary N.3.3. If $Q^{\Phi}$ is simply connected and $S$ is complete, then $f$ is an embedding, and the image of $f$ is the graph of a section of $\pi$.

A particularly suitable case is the static case, i.e., the case where $M$ contains a hypersurface $S_{0}$ everywhere orthogonal to $X$ (in other words, the case where the distribution $X^{\perp}$ is integrable). Then, the restriction to $S_{0}$ of $\pi$ is an isometric identification between $S_{0}$ and $Q^{\Phi}$. Pushing along $\Phi^{t}$, we obtain an identification between $M$ and the product $S_{0} \times \mathbb{R}$. The vector field $X$ is then $\partial / \partial t$, and the Lorentzian metric at a point $(x, t)$ is:

$$
g_{0}(x)-a(x) d t^{2}
$$

where $a(x)$ is the opposite of the norm of $X(x, t)$. Let $g_{1}$ be the metric $\frac{1}{a} g_{0}$ on $S_{0} \approx Q^{\Phi}$. Corollary N.3.3 can be formulated as follows:

Corollary N.3.4. When $X$ is static, every complete spacelike immersed surface is the graph of a 1-contracting map $s:\left(S_{0}, g_{1}\right) \rightarrow \mathbb{R}$.

In Minkowski spacetime, we obtain that complete spacelike immersed surfaces are graphs of 1-contracting maps from the Euclidean space into $\mathbb{R}$ (Mess' Lemma $1)$. In anti-de Sitter space $\operatorname{AdS} \approx \operatorname{PSL}(2, \mathbb{R})$, the right action of $\operatorname{SO}(2)$ defines a timelike isometric flow $\Phi^{t}$ for which the orbit space $\left(Q^{\Phi}, g_{0}\right)$ is isometric to the hyperbolic plane $\mathbb{H}^{2}$ (in order to be coherent with the previous presentation, we should consider the universal covering $\widetilde{\mathrm{SO}}(2)$ acting on $\widetilde{\mathrm{SL}}(2, \mathbb{R}))$. Corollary $\mathbb{N} .3 .3$ applies: see Mess' Lemma 6.

Remark. In de Sitter space, there is no everywhere-timelike Killing vector field and so we cannot use Lemma N.3.1. Indeed there are non-injective isometric immersions of complete Riemannian hypersurfaces in de Sitter space; see $\$$ N.6. 
Remark. These ideas have been extended to the situation in which $Q^{\Phi}$ is not necessarily the orbit space of a timelike Killing vector field, but only the leaf space of a 1-dimensional foliation with timelike leaves. See 62.

\section{N.4. Standard SPacetimes}

The first main result of this section is Proposition 4, which shows that the developing image of a spacelike slice is an embedding and that the time coordinate is proper. In addition, this section contains the important definition of standard spacetimes, and it is shown in Proposition 6 that a spacelike slice in an arbitrary flat spacetime admits a neighborhood that embeds in a standard spacetime.

The second half of this section handles, more or less completely, the genus 1 case: a neighborhood of a closed spacelike torus in a flat spacetime embeds in a complete spacetime or in a "standard" example (Definition 2; these are torus spacetimes in [79, 42]).

\section{N.5. Domains of DePendence AND Geodesic laminations}

This section is the heart of the paper and contains the main theorem in the flat case, which shows that domains of dependence (genus at least two) are in one-one correspondence with measured geodesic laminations.

A key idea introduced in this section is the notion of a domain of dependence. Mess provides a definition peculiar to the flat case (Definitions 3 and 4). He also mentions in the introduction a more general definition, characterizing domains of dependence as spacetimes which are maximal with respect to the property that there is a closed (compact without boundary) spacelike hypersurface through each point. It appeared to us pertinent for the reader's convenience to clarify this notion which is classical in General Relativity; this is done below in $\$$ N.5.1 and $\$$ N.5.2. Domains of dependence are maximal globally hyperbolic spacetimes (Definitions N.5.5 and N.5.11), while the definition of Mess restricts to the Cauchy compact case (see the final remark of $\$$.5.1).

The section opens with a discussion of some basic causality notions; domains of dependence are defined and Proposition 11 describes the structure of the causal horizon. (Note that several of the conclusions of Proposition 11 hold in greater generality; see for example [63, Ch. 6]). Proposition 12 is one half of the main theorem: given a hyperbolic surface and measured geodesic lamination, there is a corresponding flat spacetime. This result is a Lorentzian version of the grafting operation for complex projective surfaces, and readers unfamiliar with this construction may profit from first reading the details in [66. Mess' proof is long and contains a lot of implicit information on the structure of these examples. Proposition 13 is the other half: an arbitrary domain of dependence (in fact, its causal horizon) determines a measured geodesic lamination, inverse to the correspondence in Proposition 12. This is followed by a short interlude discussing the identification of the moduli space of domains of dependence with the (co)tangent bundle of Teichmüller space, the symplectic structure on it, and the Chern-Simons reduction due to Witten. The section concludes with Propositions 14 and 15 which show that the group action on the causal horizon is dynamically complicated; Mess uses this to characterize when domains of dependence embed in larger spacetimes. We recast this result in terms of the Cosmic Censorship Conjecture in $\$ \mathrm{N.5.3}$ below. 
The following theorem summarizes the results of $\S 5$ that lead to the parameterization in terms of measured geodesic laminations.

Theorem N.5.1. Let $M$ be a flat oriented Cauchy compact domain of dependence of dimension $2+1$. Up to a reversal of time orientation, $M$ is future complete. Moreover, $M$ is the quotient of a convex domain $D$ by a discrete group of isometries $\Gamma$. Let $g$ be the genus of the Cauchy surfaces. Then, $g$ is positive. If $g>1$, the linear part of the holonomy group $\Gamma$ is Fuchsian. If $g=1, M$ is either complete, or a Torus Universe.

For the definition of Torus Universes, see 42. In the preprint, Mess calls them "standard spacetimes" (with toral Cauchy surfaces). They are called Misner spacetimes in [17. The theorem above is generalized in [17] to higher dimensions, and by replacing the hypothesis "Cauchy compact" by the requirement that one Cauchy surface is complete.

N.5.1. Global hyperbolicity. The notion of domain of dependence is related to the causal notion of global hyperbolicity. This is essentially the strongest assumption one can make regarding the causal properties of a given spacetime; an intermediate but fundamental notion, necessary for the definition of global hyperbolicity, is strong causality. We remind the reader of the necessary definitions.

A causal (resp. timelike) curve is an immersion $c: I \subset \mathbb{R} \rightarrow M$ such that for every $t$ in $I$ the derivative $c^{\prime}(t)$ is causal (resp. timelike). This notion extends naturally to non-differentiable curves (see 24). Such a curve is extendable if there is another causal curve $\hat{c}: J \rightarrow M$ and a homeomorphism $\varphi: I \rightarrow K \subset J$ such that $K \neq J$ and $c$ coincides with $\hat{c} \circ \varphi$. The causal curve $c$ is inextendable if it is not extendable.

Remark. Spacelike hypersurfaces are locally strictly achronal; non-timelike hypersurfaces are locally achronal.

Definition N.5.2. Let $x$ and $y$ be two points in $M$, with $y$ in the future of $x$. The common past-future region $U(x, y)$ is the intersection between the past of $y$ and the future of $x$.

The domains $U(x, y)$ form the basis for a topology on $M$, the so-called Alexandrov topology (see 24]). Observe that every $U(x, y)$ is open for the manifold topology. The converse in general is false:

Definition N.5.3. If the Alexandrov topology coincides with the manifold topology, $M$ is said to be strongly causal.

Remark. If $M$ is strongly causal, every open domain $U \subset M$ equipped with the restriction of the ambient Lorentzian metric is strongly causal.

Proposition N.5.4 (Proposition 3.11 of 24]). A Lorentzian manifold $M$ is strongly causal if and only if it satisfies the following property: for every point $x$ in $M$, every neighborhood of $x$ contains an open neighborhood $U$ (for the usual manifold topology) which is causally convex, i.e., such that any timelike curve in $M$ joining two points in $U$ is actually contained in $U$.

Definition N.5.5 (24, p. 11). $M$ is globally hyperbolic if:

- it is strongly causal, 
- for any $x, y$ in $M$, the intersection between the causal future of $x$ and the causal past of $y$ is compact.

From now on, we assume that $M$ is strongly causal.

The notion of global hyperbolicity is closely related to the notion of Cauchy surfaces: let $S$ be a spacelike surface embedded in $M$.

Definition N.5.6. The past development $P(S)$ (resp. the future development $F(S))$ is the set of points $x$ in $M$ such that every inextendable causal path containing $x$ meets $S$ in its future (resp. in its past). The Cauchy development $\mathcal{C}(S)$ is the union $P(S) \cup F(S)$.

Definition N.5.7. If $S$ is acausal and $\mathcal{C}(S)$ is all of $M$, then $S$ is said to be a Cauchy surface.

Theorem N.5.8 ([57]). A Lorentzian manifold $M$ is globally hyperbolic if and only if it admits a Cauchy surface.

Theorem N.5.9 ([57], Proposition 6.6.8 of [63]). If $M$ is globally hyperbolic, and $S$ is a Cauchy surface of $M$, there is a diffeomorphism $f: M \rightarrow S \times \mathbb{R}$ such that every $f^{-1}(S \times\{*\})$ is a Cauchy surface in $M$.

Remark. There has been some imprecision in the literature concerning the proof of the smoothness of the splitting of globally hyperbolic spacetimes. See [27, 28, 29] for a survey of this question, and a complete proof of the smoothness of the splitting $M \approx S \times \mathbb{R}$.

Remark. A globally hyperbolic spacetime is said to be Cauchy compact (or spatially compact) if it admits a closed Cauchy hypersurface. All the Cauchy hypersurfaces are then closed, and every closed embedded spacelike hypersurface is a Cauchy hypersurface. The (well-known) proofs of these assertions are as follows: if $S^{\prime} \subset M \approx S \times \mathbb{R}$ is a (connected) closed spacelike hypersurface, the projection on the first factor $S$ induces a local homeomorphism $p: S^{\prime} \rightarrow S$. The compactness implies that $p$ is a covering map. The chronological orientation induces an ordering on the fibers of $p$ which is preserved by lifting of curves. It follows that this covering is trivial, i.e., a homeomorphism. Hence, $S^{\prime}$ is the graph of some function $f: S \rightarrow \mathbb{R}$. Let $t: M \rightarrow \mathbb{R}$ the projection on the second factor. Since $S$ is compact, $t \circ f$ has bounded image. Since $t$ is strictly increasing from $-\infty$ to $+\infty$ along every inextendable causal curve, it follows that $S^{\prime}$ is a Cauchy hypersurface.

\section{N.5.2. Maximal globally hyperbolic spacetimes.}

Definition N.5.10. An isometric embedding $f: M \rightarrow N$ is a Cauchy embedding if the image under $f$ of any Cauchy surface in $M$ is a Cauchy surface of $N$.

Definition N.5.11 ([63, pp. 251-252). A globally hyperbolic spacetime $M$ is maximal if every Cauchy embedding $f: M \rightarrow N$ in a spacetime with constant curvature is surjective.

Theorem N.5.12 (see Choquet-Bruhat-Geroch [44]). Let $M$ be a globally hyperbolic spacetime with constant curvature. Then there is a Cauchy embedding $f: M \rightarrow N$ in a maximal globally hyperbolic spacetime $N$ with constant curvature. Moreover, this maximal globally hyperbolic extension is unique up to right composition by an isometry. 
Remark. Actually, this theorem admits a much more general extension: instead of restricting to spacetimes with constant curvature, we need only require that the spacetimes under consideration satisfy the Einstein equation (in the void, with cosmological constant).

Remark. The notion of global hyperbolicity is linked with the most usual way to find solutions of the Einstein equation: i.e., solving the associated Cauchy problem. This approach consists of considering a manifold $\Sigma$ endowed with a Riemannian metric $\bar{g}$ and a symmetric 2-tensor $I I$, and trying to find a Lorentzian metric $g$ on $M=\Sigma \times(-1,+1)$, such that $g$ satisfies the Einstein equation, such that $\bar{g}$ is the restriction of $g$ on $\Sigma=\Sigma \times\{0\}$ and such that $I I$ represents the second fundamental form of $\Sigma=\Sigma \times\{0\}$ in $M=\Sigma \times(-1,+1)$. For the problem to admit a solution, the initial data $(\Sigma, \bar{g}, I I)$ must satisfy the constraint equations (for geometers, the contracted Gauss-Codazzi equations). Conversely, the Choquet-Bruhat theorem ([50]) states that every set of initial data satisfying the constraint equations leads to a solution, which, by the nature of the process, is globally hyperbolic. Furthermore, according to the Choquet-Bruhat-Geroch Theorem (N.5.12) above, there is a unique maximal globally hyperbolic solution (up to isometry).

N.5.3. Cosmic Censorship. Let's reproduce here part of the content of Proposition 15:

Proposition N.5.13. Let $M$ be a flat domain of dependence of dimension $2+1$. Assume that the holonomy of $M$ fixes a point in Minkowski space, or that the associated measured geodesic lamination has no isolated leaf. Then, $M$ is a maximal flat spacetime, i.e., any isometric embedding $f: M \rightarrow N$ in a flat spacetime $N$ (even not globally hyperbolic) is surjective.

The proof of this proposition rests on the study of the dynamical properties of the group action on the boundary of $D$, where $D$ is the convex domain in Minkowski space such that $M=\Gamma \backslash D$. Actually, nearly the same proof shows that this proposition holds also for anti-de Sitter or de Sitter domains of dependence (see [89, Ch.10]).

This proposition has a definite physical flavour: indeed, it resolves a special case of the Strong Cosmic Censorship Conjecture:

Strong Cosmic Censorship Conjecture [86]: Let $M$ be a compact manifold. Then, for every set of generic Cauchy data $(M, \bar{g})$, the maximal globally hyperbolic domain of dependence defined by these data is a maximal spacetime in the $C^{2}$ category.

Since measured geodesic laminations without isolated leaves are dense among measured geodesic laminations, Proposition N.5.13 is essentially the proof of Cosmic Censorship in the flat $2+1$-dimensional case (modulo the verification that the map associating a measured geodesic lamination to Cauchy data is open - this fact does not appear in Mess' paper, nor has a proof been given elsewhere in the literature to the best of our knowledge). The hypothesis "without isolated leaves" is essential, since, as explained by Mess, it is easy to extend the domain of dependence outside $D$ if the measured geodesic lamination contains an isolated leaf. This is one way to see that the Cosmic Censorship Conjecture fails if the "generic" requirement is dropped.

For more details, see $\S 2.1$ of $[5]$. 
Remark. The content of the paper [65] is the proof of Proposition N.5.13 for the flat case in any dimension, but only in the case where the holonomy preserves a point in Minkowski space. This case follows actually quite easily from the minimality of strong stable foliations of geodesic flows of negatively curved Riemannian manifolds: this is precisely what is re-proved in 65].

N.5.4. CMC time. In the introduction, Mess indicates that he has nothing to say about "the interesting question of foliating the manifolds we consider by surfaces of constant mean curvature [...]". At the end of $\S 5$, he mentions this problem once again, cites the work of Moncrief [84, and leaves the problem of existence and uniqueness of such a foliation as a question. This question has been answered recently; see [18, [19], and 20] for a sketchy proof in the de Sitter case. We summarize these results here, postponing a more detailed discussion of CMC foliations and their asymptotics (focusing on the flat case) to $\$$ N.10

Definition N.5.14. A function $t: M \rightarrow \mathbb{R}$ on a domain of dependence $M$ is a CMC time if $t$ is a time function, i.e., strictly increasing along every causal curve, and every fiber $t^{-1}(s)$ is a spacelike hypersurface with constant mean curvature $s$.

Remark. The sign of the mean curvature is important. The convention for defining the second fundamental form of $S_{s}=t^{-1}(s)$ is to take as normal vector field along $S_{s}$ the future-oriented one. It follows from the maximum principle that, for a given spacetime $M$, if a CMC time function exists, then it is unique, and is as regular as the Lorentzian manifold $M$. See [19] for more details.

Theorem N.5.15 (Theorem 12.1 in [17; see also [4). Every flat future-complete Cauchy-compact domain of dependence $M$ admits a CMC time function $t: M \rightarrow$ $(-\infty, 0)$, except if $M$ is complete.

The de Sitter case is also valid (see [90] for the definition of parabolic de Sitter domains of dependence).

Theorem N.5.16 (Theorem 5.4 in [20]). Every future-complete Cauchy-compact domain of dependence of constant positive curvature and dimension $2+1$ admits a CMC time function $t: M \rightarrow(-\infty,-2)$, except if $M$ is complete or parabolic.

The anti-de Sitter case is the easiest to formulate:

Theorem N.5.17 (19]). Every Cauchy-compact domain of dependence of constant negative curvature and dimension $2+1$ admits a CMC time function $t: M \rightarrow$ $(-\infty,+\infty)$

\section{N.6. The CASE OF DE Sitter SPACE}

In $\S 6$, Mess constructs standard de Sitter spacetimes $S \times \mathbb{R}$ in terms of (complex) projective structures on $S$ (Proposition 16). The corresponding classification theorem (that every de Sitter domain of dependence comes from this construction) is only proved in special cases: $1+1$-dimensional de Sitter spacetimes in Proposition 17 and the genus one $2+1$-dimensional case in Proposition 18 .

There are two standard parameterizations of the space $P(S)$ of projective structures on $S$; the first is the by the bundle of holomorphic quadratic differentials over Teichmüller space, where a quadratic differential comes from the Schwarzian derivative of the developing map of a projective structure. Mess introduces these notions briefly, and shows how the Bers embedding gives a holomorphic section of 
the bundle $P(S) \rightarrow$ Teich $(S)$. His assertion that the holonomy map from $P(S)$ to the $P S L(2, \mathbb{C})$ representation variety is a holomorphic submersion is due to Hejhal 64.

The second parameterization was given by Thurston and is the one used by Mess in the construction of the standard de Sitter examples. The key element in the Thurston parameterization is the grafting operation, which produces a new projective structure on $S$ from a given hyperbolic structure and measured lamination. In the simplest case that the measured lamination is a simple closed curve $\gamma$ with weight $t>0$, grafting amounts to inserting a projective annulus along $\gamma$ of height $t$. This extends by continuity to any measured lamination, defining a map

$$
\Theta: \operatorname{Teich}(S) \times \mathrm{ML} \rightarrow P(S),
$$

which turns out to be a homeomorphism (an exposition of Thurston's proof is given in 66).

One way to obtain the inverse $\Theta^{-1}$ is by "thickening" the developing map $d$ : $\tilde{S} \rightarrow \mathbb{C P}^{1}$ of a projective structure to an immersion $D: \tilde{S} \times(0, \infty) \rightarrow \mathbb{H}^{3}$ equivariant with respect to the holonomy in $\operatorname{PSL}(2, \mathbb{C}) \cong \operatorname{Isom}^{+}\left(\mathbb{H}^{3}\right)$. The measured geodesic lamination is then obtained as a bending lamination on the frontier of the image of $D$, where some care is required if $d$ is not an embedding.

After some introductory material on the Klein model of de Sitter space and projective structures, Mess gives the construction of $D$ in terms of so-called maximal balls in the universal cover $\tilde{S}$. There are some minor inaccuracies in this discussion, such as the assertion that the curves foliating overlapping maximal balls develop to circular arcs in $\mathbb{C P}^{1}$ with the same endpoints. We recommend Kulkarni and Pinkall's later paper [72] as the best place to read the details of the maximal ball construction (the cited paper [71] is quite different). Also note that the reference to [10] was probably intended to have been one of Apanasov's later papers, e.g. 11].

Then, the projective dual of the map $D$ is used to produce an equivariant immersion $q: \tilde{S} \times(0, \infty) \rightarrow \mathbb{S}_{1}^{3}$. These are the standard de Sitter spacetimes, and their basic properties are given in Proposition 16. Mess conjectures that every $2+1$-dimensional de Sitter spacetime which is a small neighborhood of a closed oriented spacelike surface embeds in a unique standard spacetime. This was shown by Scannell in his thesis 89,90 .

It is well-known that the developing map of a projective structure on a surface need not be an embedding or even a covering of its image [78, and so the same is true for the developing maps of standard de Sitter spacetimes. This is in contrast to the flat and anti-de Sitter cases (as discussed in $\$ \mathrm{~N} .3$ above), and represents the primary difficulty in extending the classification theorems to the de Sitter case. This is overcome by defining domains of dependence, etc., solely in terms of the causal structure induced by the developing map, and not in terms of the geometry of de Sitter space itself.

The maximal ball construction for projective surfaces works in any dimension when a manifold is equipped with a flat conformal (Möbius) structure [72]. It turns out that the classification theorem extends as well:

Theorem N.6.1. 90 Let $M$ be a closed n-manifold. Then there is a bijective correspondence between the moduli space of flat conformal structures on $M$ and the moduli space of maximal $n+1$-dimensional de Sitter domains of dependence homeomorphic to $M \times \mathbb{R}$. 
The second half of $\S 6$ in Mess' paper contains an approach to his conjecture. The outline he proposes is completely different than the proof eventually given by Scannell, and it would be interesting to know if it can be made to work, despite being special to the $2+1$-dimensional case. More precisely, if one has a (time-oriented) $2+1$-de Sitter spacetime which is a neighborhood of a closed oriented spacelike surface $S$, then there is an associated holonomy representation hol : $\pi_{1}(S) \rightarrow$ $P S L(2, \mathbb{C})$. To show the spacetime comes from a projective structure on $S$ via the construction above, it would help to know that hol in fact coincides with the holonomy of some projective structure. For this, Mess appeals to the following famous "prescribed monodromy" result:

Theorem N.6.2. Let $S$ be a closed oriented surface of genus at least two, and let $h: \pi_{1}(S) \rightarrow P S L(2, \mathbb{C})$ be a homomorphism. Then $h$ is the holonomy representation of some complex projective structure on $S$ if and only if $(1)$ h lifts to $S L(2, \mathbb{C})$ and (2) $h$ is non-elementary.

Mess cites Gallo's announcement [52] for this, though the complete proof was not made available until several years later, in joint work with Marden and M. Kapovich [54. Gallo's proposed proof of Theorem N.6.2 is based on the existence of a pants decomposition of $S$ with the property that the holonomy of each pair of pants is quasi-Fuchsian. Mess argues from this that (after a small change in the holonomy) one can assume that the holonomy of each pair of pants is Fuchsian. But even with this assumption it is not clear how to produce a projective structure on $S$ without appealing to the more general convexity results in [90]. There is also an issue with changing the holonomy, as is pointed out in the proof of Proposition 18.

In any case, Mess succeeds in proving the conjecture for $1+1$-dimensional spacetimes (Proposition 17) and genus one $2+1$-dimensional spacetimes (Proposition 18).

Remark. Tan's UCLA thesis [95] and a (never published) preprint of Gallo, Goldman, and Porter 53] are cited for the classification of projective structures with holonomy in $P G L(2, \mathbb{R})$; for the former, see [96] and for the latter [54] (cf. [58]).

\section{N.7. Anti-De SitTer MANifolds}

In $\S 7$, Mess proves a classification theorem for anti-de Sitter spacetimes that is analogous to the classification in the flat case; we summarize this as follows:

Theorem N.7.1. Let $M$ be an oriented Cauchy compact domain of dependence locally modeled on anti-de Sitter space. Then, $M$ is the quotient of a convex domain $H$ of $A d S$ by a discrete group of isometries $\Gamma$. The genus $g$ of the Cauchy surfaces is positive, and if $g>1$, the holonomy morphism $\rho=\left(\rho_{L}, \rho_{R}\right) \rightarrow \operatorname{PSL}(2, \mathbb{R}) \times$ $\operatorname{PSL}(2, \mathbb{R})$ is a pair of Fuchsian representations. All pairs $\left(\rho_{L}, \rho_{R}\right)$ of Fuchsian representations are realized.

In the second half of the section (immediately following Proposition 21) Mess recasts these results in the language of earthquakes, observing that they give an alternative proof of Thurston's theorem that any two points of Teichmüller space differ by a left earthquake.

The section opens with a discussion of the projective model of anti-de Sitter space and identifies the identity component of its isometry group as $P S L(2, \mathbb{R}) \times$ 
$P S L(2, \mathbb{R})$. Proposition 19 shows that the holonomy of a closed spacelike surface (implicitly with genus $g>1$ ) in an anti-de Sitter spacetime maps to a Fuchsian group in each factor. (The proof is analogous to Proposition 1 for the flat case, by arguing that the Euler classes must be $2-2 g$ and then appealing to Goldman's Theorem). Thus the holonomy determines a pair $(x, y)$ in $\operatorname{Teich}(S) \times \operatorname{Teich}(S)$, and the left and right representations are conjugated by some homeomorphism $h: \mathbb{R P}^{1} \rightarrow \mathbb{R P}^{1}$. The graph of $h$ is a closed achronal topological circle at infinity. The convex hull $X(\phi)$ of this graph quotiented by the holonomy is an anti-de Sitter spacetime $X(x, y)$.

The existence of the spacetime $X(x, y)$ is the main part of Proposition 20. It is also claimed that $X(x, y)$ is uniquely determined by $x, y$, and the fact that its boundary is spacelike, locally convex and without extreme points. No proof is given however, with the discussion of Proposition 20 ending abruptly with the statement "Now for the uniqueness of $X(\phi)$ " before Lemma 6.

Let $S$ be a closed spacelike surface in a spacetime $M$ locally modeled on AdS. Then, the inclusion $S \subset M$ is incompressible, and the restriction to $\widetilde{S}$ (a lift in $\widetilde{M}$ of $S$ ) of $\mathcal{D}$ is injective (see Lemma 6 and compare Lemma 1 from the flat case). These statements follow from the arguments given above in $\$ \mathrm{~N} .3$. But something more is needed (given by Mess as Lemma 7 ): the restriction of $\mathcal{D}$ to $\widetilde{S} \approx \mathbb{D}^{2}$ extends to a continuous map $\overline{\mathcal{D}}$ from the closed disc $\overline{\mathbb{D}}^{2}$ into the closure of AdS $\approx X$ in $\mathbb{R P}^{3}$, such that the image by $\overline{\mathcal{D}}$ of the boundary $\partial \mathbb{D}^{2}$ is a topological circle in the hyperboloid $Q$. Mess proposes two sophisticated proofs of this statement. Actually, there is a very simple proof of this fact, using the natural conformal embedding of AdS into $\mathbb{S}^{2} \times \mathbb{S}^{1}$ : see [19] or [15].

During the proof of Lemma 7, Mess shows that the Cauchy development $H(A)$ of $A$ only depends on $A_{\infty}$ : write it as $H\left(A_{\infty}\right)$. This leads to Proposition 21: any closed spacelike surface $S$ in an anti-de Sitter spacetime admits a neighborhood which can be embedded in a domain of dependence $\Gamma \backslash H\left(A_{\infty}\right)$.

Actually, Proposition 21 starts with a claim that the genus of such a surface has to be greater than one. This claim is false: there are indeed anti-de Sitter domains of dependence with toral Cauchy surfaces: the so-called Torus universes (which seem to have been first defined in [79]; see also [42, [15], and the last section of [19]). Mess proposed two "proofs" for this statement. The first: "We could argue that the holonomy cover $\hat{F}$ is quasi-isometric to a hyperbolic plane, and so the ball of radius $r$ in $\hat{F}$ grows exponentially with $r$, while the cover of a torus has only polynomial volume growth". But he doesn't argue more. "Alternatively, the left holonomy defines a homomorphism from $\mathbb{Z} \oplus \mathbb{Z}$ into an abelian subgroup [...] (with, after perturbation) cyclic image. The right holonomy is topologically conjugate to the left holonomy so the holonomy has kernel which contradicts lemma 6.". The error is that left and right holonomies are not conjugate, only semi-conjugate; the proof of the conjugacy involved Goldman's theorem proving the representations into $\operatorname{PSL}(2, \mathbb{R})$ with maximal Euler number are all Fuchsian, and hence is not valid for $g=1$. Actually, when $g=1$, the topological circle $A_{\infty}$ is a union of four lightlike segments. It can be interpreted as the graph of a semi-conjugacy, shrinking two intervals of $\mathbb{R P}^{1}$ to two points, and expanding the two common extremities of these intervals to intervals.

As noted earlier, the second half of $\S 7$ reinterprets the geometric data on the boundary of the convex hull $X(\phi)$ as earthquakes, reproving Thurston's Earthquake 
Theorem. Then, Mess considers the case where $S$ is not compact. He considers the example of an earthquake "taking a complete hyperbolic surface with cyclic fundamental group and one cusp to one of the components of the complement of a closed geodesic in a hyperbolic surface with cyclic fundamental group and no cusp". This example corresponds to extreme black-holes as described in [16. He also considers the example of the thrice punctured sphere, which is studied in more detail in 25.

The section closes with a number of interesting questions: the volumes of the domain of dependence and of the convex hull define two maps on $\operatorname{Teich}(S) \times \operatorname{Teich}(S)$. "How do they behave? Are they related, perhaps asymptotically, to such invariants of a quasi-Fuchsian groups as the volume of the convex hull and the Hausdorff dimension of the limit set?". It is worth noting that the meaning of this question can be clarified thanks to Benedetti-Bonsante Wick rotation (see the penultimate remark in $\$ \mathrm{~N} .9$ and also $\S 4.8 .2$ of $[25$. for specific computations in this direction).

Mess then asks several questions about the geometry of the boundary of the convex core in both the anti-de Sitter and hyperbolic settings. Concerning the problem of prescribing the induced metric on the boundary of the convex core, in the hyperbolic setting, he mentions that existence follows from work of Epstein and Marden. This existence statement is also a direct consequence of a result of Labourie [73].

As for prescribing the bending lamination, still in the hyperbolic setting, the existence has been proved recently by Bonahon and Otal 33 for convex co-compact manifolds with incompressible boundary (and, for general convex co-compact manifolds, by Lecuire [76]). However, for prescribing either the induced metric or the bending lamination, the uniqueness remains unknown, basically because we do not know whether infinitesimal rigidity holds, i.e. whether any first-order deformation of a quasi-Fuchsian manifold induces a non-zero variation of the induced metric (resp. bending lamination) on the boundary of the convex core. However, in the hyperbolic setting, Bonahon [30] proved that the two infinitesimal rigidity questions, concerning the induced metric and concerning the bending lamination, are equivalent. Bonahon [31] also gave a careful analysis of what happens near the "Fuchsian locus", showing that uniqueness does hold there. Series 94 proves uniqueness in the case of once-punctured tori.

Mess also asks whether it is possible to prescribe the induced metric on one boundary component, and the bending lamination on the other. Lecuire [77] has recently proved the existence part of this statement (again in the hyperbolic case). In the same preprint he also gives a positive answer to the existence part of another of the questions asked by Mess: whether it is possible to prescribe the conformal structure on the upper surface at infinity and the measured bending lamination on the lower boundary of the convex core; the uniqueness holds when the prescribed bending lamination is supported on simple closed curves. The first result is largely a consequence of the second, which is proved using recent results of Bromberg [40] on the deformations of complete hyperbolic metrics with cone singularities along closed curves.

Mess also asks whether a quasifuchsian hyperbolic manifold is uniquely determined by the conformal structure on the upper surface at infinity along with the induced metric on the lower boundary of the convex core. Here again, the existence should hold, and should follow directly from the argument that he mentions 
above, using the fact that the induced metric on the lower boundary of the convex core is quasi-conformal to the conformal structure on the lower surface at infinity (according to [4]).

Another known result in this same vein is that one can prescribe a conformal structure at infinity and the bending lamination associated to that conformal structure and these data determine the quasi-Fuchsian group uniquely; see 92 .

The article goes on to ask similar questions in the anti-de Sitter setting. The questions on prescribing the induced metric and/or the measured bending lamination on the boundary of the convex core remain open. However the arguments given in section 7 give an answer to one of the questions: whether a maximal globally hyperbolic AdS manifold is uniquely determined by its left holonomy (say $h_{l}$ ) and the induced metric on the upper boundary of its convex core. By Thurston's earthquake theorem, there is a unique measured lamination $\lambda_{+}$such that $h_{l}$ is the image of $\mu_{+}$by the left earthquake along $\lambda_{+}$. The right holonomy $h_{r}$ is then the image of $\mu_{+}$by the right earthquake along $\lambda_{+}$. A similar argument also shows that a maximal globally hyperbolic AdS manifold is uniquely determined by the left holonomy and the measured bending lamination on the upper boundary of the convex core.

It is perhaps worth remarking that one can replace in these questions the convex core by a slightly larger domain, with smooth and strictly convex boundary. In the hyperbolic setting, the induced metric now has curvature $K>-1$, and it is actually possible to obtain any pair of such metrics uniquely (see 93). For the same convex domains the smooth analogue of the bending lamination is the third fundamental form, which is a smooth metric with $K<1$ and contractible closed geodesics of length larger than $2 \pi$, and, here again, any pair of such metrics can be uniquely prescribed (also 93 ).

The anti-de Sitter case remains more elusive than the quasi-Fuchsian case, though some of the results known in the hyperbolic setting might extend to anti-de Sitter manifolds. In particular, this is the case for the analysis of [31, and also for 93. (for which however the proof has to be adapted and the dictionary between quasi-Fuchsian and anti-de Sitter manifolds has to be extended a little).

Remark. Theorem N.7.1 has been extended recently (in 25] and [15]) to the case of domains of dependence of dimension $2+1$ admitting complete Cauchy surfaces. But the classification of Cauchy compact domains of dependence in higher dimensions remains an open problem.

Remark. Another proof that the moduli space of anti-de Sitter domains of dependence is parameterized by two copies of Teichmüller space is given in [70. This work is based on differential-geometric ideas and uses a result of Labourie 74 on mappings between hyperbolic surfaces.

Remark. A striking fact is that geometric ideas presented in Mess' preprint provide an essential complement to the abundant literature devoted to BTZ (multi) blackholes (see 14, 13, 3, 1, 2, 38, 39, 42, etc... This matter is developed in 15, 16.

Remark. The analogy between representations of surface groups in the isometry group of anti-de Sitter space and quasi-Fuchsian groups has been pursued and generalized in recent work of Labourie, in which a definition of a quasi-Fuchsian group in a general Lie group $G$ is given. Such a notion is characterized by the choice of a specific $H=S L(2, \mathbb{R})$ in $G$. Surface groups which factor through a 
cocompact subgroup of $H$ are called Fuchsian. Then quasi-Fuchsian (or Anosov) subgroups are deformations of these subgroups which satisfy a dynamical condition, and the classical stability theorem of dynamical systems ensures that the space of quasi-Fuchsian representations is open. It follows from the definition that a quasiFuchsian group generates a continuous limit curve at a suitable infinity which is a Hölder equivariant mapping from the boundary at infinity of the surface to a parabolic quotient. One may make the link with the situation described by Mess following Proposition 19, which gives the curve at infinity in $\mathbb{R} \mathbb{P}^{1} \times \mathbb{R} \mathbb{P}^{1}$.

It turns out that two classes of Lie groups have some special and interesting features in this context: real split groups and isometry groups of hermitian symmetric spaces of tube type. In these cases, the limit curve enjoys an extra positivity condition (see Fock-Goncharov [49], and Burger-Iozzi-Labourie-Wienhard [41]) which, in the case of $S O(2,2)$ (which belongs to both classes), amounts to the condition that it be a spacelike curve. In both cases, it also turns out that quasi-Fuchsian groups fill out a whole component of the space of representations.

Remark. The ideas involved in Mess' proof of the Earthquake Theorem have been extended recently to two contexts. In [37, the 3-manifolds considered are AdS manifolds with "particles" - cone singularities along maximal time-like lines, with angle less than $\pi$ - but which are globally hyperbolic. These manifolds happen to have a well-defined convex core, which leads to a version of the Earthquake Theorem for hyperbolic surfaces with cone singularities of angle less than $\pi$. In [36], the same idea is used for multi-black holes, which are not globally hyperbolic. These manifolds also have a kind of convex core, and considering its geometry leads to an extension of the Earthquake Theorem for hyperbolic surfaces with geodesic boundary: the measured laminations on the interior of those surfaces act simply transitively by right earthquakes on the enhanced Teichmüller space. The enhanced Teichmüller space - recently introduced by Fock - contains an open dense subset which is a $2^{n}$-cover of the "usual" Teichmüller space, where $n$ is the number of boundary components.

\section{N.8. Classification of spacetimes}

This section starts with a report on results about complete flat Lorentzian manifolds, in particular the proof (Proposition 24) that the linear holonomy is either solvable or discrete. This result appeared earlier (with a different proof that appeals to 12]) in Fried-Goldman [51.

This is followed by "Mess' Theorem" (Proposition 25): the fundamental group of a closed surface cannot act properly on all of Minkowski space. The proof essentially relies on the "Cosmic Censorship" machinery from $\S 5$. New proofs have been given recently by Goldman-Margulis 60, and Labourie [75.

A consequence of this result is that the fundamental group of a complete, noncompact, flat Lorentz 3-manifold is solvable or free. Mess goes on to say that "it seems plausible that a complete Lorentz manifold with free fundamental group is diffeomorphic to the interior of a (possibly non-orientable) handlebody", but this question remains open. On the other hand, the cited conjecture of Margulis that a complete Lorentz manifold with finitely generated free fundametal group has discrete and purely hyperbolic linear holonomy has been disproven; Drumm [45] constructs affine deformations of any free Fuchsian group which act properly on Minkowski space. 
At the heart of the Goldman-Margulis proof of Mess' Theorem is the result that the Margulis signed length (see [60]) is the Hamiltonian flow of the length in Teichmüller space; this is conjectured by Mess (and "left to the reader") in the extended discussion of quantization and Teichmüller theory following Proposition 25. Quantization of Teichmüller spaces has become a classical theme in the last several years. The initial breakthrough was due to Chekhov and Fock [48, who quantized the algebra of observables given by the shear coordinates on open surfaces. By developing this technology, invariants of hyperbolic 3-manifolds and more generally of 3-manifolds equipped with $P S L(2, \mathbb{C})$ characters have been constructed (see Kashaev [67, followed by works of Baseilhac and Benedetti [21], 22] and Bonahon and Liu [32]). As yet, however, the relationship between anti-de Sitter and physical $2+1$ gravity is not completely clear.

Mess' suspicion that the spectrum of the Weil-Petersson Laplacian on moduli space is bounded away from zero has been confirmed by McMullen (in his paper showing moduli space is Kähler hyperbolic [81]).

Next Mess proves the following theorem (Proposition 26):

Theorem N.8.1. Let $M$ be a compact flat $2+1$ spacetime with spacelike boundary (maybe empty). Then, either $M$ is complete (if the boundary is empty), or diffeomorphic to $S \times[0,1]$ where every $S \times\{t\}$ is spacelike.

Actually, Mess' proof implies slightly more: in the non-closed case, the interior of $M$ is globally hyperbolic. This theorem is a natural extension of Carrière's Theorem establishing the completeness of closed flat spacetimes (43]).

It seems possible to simplify the proof by using the cosmological time (see [7]): once one has obtained by Koszul's argument that (in Mess' notation) $\hat{M}^{\prime}$ is the quotient of an open domain, then one can show that if $M$ is not complete, then the cosmological time of $M^{\prime}$ is regular (up to time reversing); it follows directly from 7] that $M^{\prime}$ is globally hyperbolic.

This result is followed by an analogous statement concerning time-oriented locally anti-de Sitter compact spacetimes with spacelike boundary (Proposition 27). Observe that the conclusion of the last statement of this proposition can be greatly simplified by the result of Klingler [68]: these spacetimes are either globally hyperbolic (Mess says that "they embed in a domain of dependence") or complete (since according to 68, closed anti-de Sitter spacetimes are complete). Indeed, the last two pages of the proof concern the closed case and can therefore be skipped in favor of 68. Observe also that the proof has to be corrected, since Mess starts the proof arguing that the boundary components have genus greater than one according to Proposition 21, and we have seen that this is not correct.

Theorem N.8.1 implies that the interior of a compact flat $2+1$-spacetime is globally hyperbolic. The analogous statement for anti-de Sitter spacetimes holds when the boundary is non-empty, but is false in the closed case: indeed, quotients of the entire anti-de Sitter space are never globally hyperbolic.

Remark. In order to conclude the case with boundary, Mess argues that the action of the surface group on a connected component of the horizon of a domain of dependence in AdS is topologically conjugate to the action on the horizon of a flat domain of dependence to which the "Cosmic Censorship" principle applies. This remark is a precursor to the "canonical Wick rotation" discussed in $\$$ N.9. 
Mess concludes with some comments on the anti-de Sitter case which are no longer relevant in light of Klingler's Theorem; later work on the classification of closed anti-de Sitter spacetimes can be found in [87, 88], and [98. The questions posed concerning de Sitter spacetimes have also been resolved; Klingler's result 68] (see also [85]) implies that there can be no closed de Sitter manifold (any dimension) as predicted by Mess. The bounded case is a bit more delicate; Scannell's thesis [89] gives necessary and sufficient conditions for a $2+1$-dimensional de Sitter domain of dependence to be maximal. There are even counterexamples to the statement given by Mess in the 1+1-dimensional de Sitter case [90, Figures 1 and 2].

At the very end, Mess mentions the higher dimensional (flat) case. He provides an argument proving that the holonomy of spacelike hypersurfaces is always discrete: this is what we explain here in \$N.4. In the flat case, it allows one to avoid the use of Goldman's Theorem: this is detailed in [17]. Indeed, the final claim of the paper about the flat $3+1$ dimensional case can also be found in [17].

\section{N.9. Canonical Wick Rotation}

Given an orientable surface $S$ let us denote by $\mathcal{M S}_{k}(S)$ the space of Lorentzian structures on $S \times \mathbb{R}$ of constant curvature $k$ such that $S \times\{0\}$ is a complete Cauchy surface up to the action of the homotopically trivial diffeomorphisms fixing $S \times\{0\}$.

When $S$ is compact, the space $\mathcal{M S}_{k}(S)$ has been shown to be homeomorphic to the cotangent bundle of the Teichmüller space of $S$, provided that the genus of $S$ is at least 2 (by Mess in the case $k \leq 0$ and Scannell 90 in the case $k=1$ ).

In [26], Benedetti and Guadagnini stressed the role of cosmological time as a fundamental tool to better understand flat globally hyperbolic spacetimes classified by Mess. In fact, the cosmological time turns out to be an important object also in [90. A remarkable fact is that, in both contexts, level surfaces of the cosmological time are obtained by grafting a hyperbolic surface $F$ (homeomorphic to $S$ ) along a measured geodesic lamination $\lambda$. Moreover, the Mess parameters are explicitly related to the pair $(F, \lambda)$ (actually they furnish good parameters for the space $\left.\mathcal{M S}_{k}(S)\right)$.

Similar behavior occurs in the anti-de Sitter framework, even if in this case cosmological time is a $\mathrm{C}^{1,1}$-function until it reaches the value $\pi / 2$. Anyway, also in this case level surfaces for values $<\pi / 2$ are obtained by grafting a hyperbolic surface along a measured geodesic lamination, and these data determine the spacetime.

When $S$ is not compact, one could try to generalize the parameterizations of $\mathcal{M S}_{k}(S)$ and $P(S)$. In fact, the notion of measured geodesic lamination can be implemented for every hyperbolic surface, and it is not difficult to see that the Mess and Thurston constructions work as well. But in this case they do not give rise to a complete classification of $\mathcal{M S}_{k}(S)$ or $P(S)$ (i.e., there are globally hyperbolic spacetimes of constant curvature such that the cosmological level sets are not obtained by grafting a hyperbolic surface along a measured geodesic lamination).

There are two natural problems arising from this remark:

1) To find a more general notion of measured geodesic lamination, coinciding with the usual one in the compact case, that allows one to generalize the Mess and Thurston constructions to obtain complete classifications of $\mathcal{M S}_{k}(S)$ as well as $P(S)$. 
2) To make explicit the identifications between $P(S)$ and $\mathcal{M S}_{k}(S)$ for compact $S$ (arising from the Thurston and Mess parameterizations) in order to see whether they can be generalized to the non-compact case.

In [72, Kulkarni and Pinkall introduced the notion of a measured geodesic lamination on a straight convex set that allows one to carry out a complete classification of projective structures on a surface $S$ with non-abelian fundamental group. Actually, they showed that the Thurston construction could be applied to these more general laminations and that every projective structure could be constructed in such a way.

In 25, it is shown that the Mess constructions could be applied also to these laminations and this leads to a complete classification of $\mathcal{M S}_{k}(S)$.

In the flat case, the proof is based on [17, 4, which provide a clear picture of the universal covering spaces and the linear holonomies of globally hyperbolic flat spacetimes. On the other hand, in 35 the universal covering spaces are classified in terms of measured geodesic laminations on straight convex sets.

The proofs in the de Sitter and the anti-de Sitter cases are carried over in [25] by developing the ideas of Mess and Scannell in this more general case, and by using an explicit map

$$
\mathcal{M S}_{0}(S) \rightarrow \mathcal{M S}_{k}(S)
$$

that solves question 2) above. In fact, such a map is constructed by developing a canonical Wick rotation and rescaling theory.

Let us briefly introduce these notions. In general, given a manifold $M$, a nowhere vanishing vector field $X$, and a pair of positive functions $\alpha, \beta$, the Wick rotation is an operation transforming Riemannian metrics on $M$ into Lorentzian metrics that make $X$ a timelike vector field. Namely, given a Riemannian metric $g$ the metric $h=W_{(X, \alpha, \beta)}(g)$ obtained by the Wick rotation of $g$ along $X$ with rescaling functions $\alpha$ and $\beta$ is determined by the following properties:

1. $X^{\perp_{g}}=X^{\perp_{h}}=X^{\perp}$.

2. $\left.h\right|_{X^{\perp}}=\left.\alpha g\right|_{X^{\perp}}$.

3. $h(X, X)=-\beta g(X, X)$.

Clearly, the Wick rotation can also be regarded as an operation transforming Lorentzian metrics with $X$ a timelike vector field into Riemannian metrics.

The rescaling operation similarly depends on a vector field $X$ and two positive functions $\alpha, \beta$, and acts on the space of Lorentzian metrics that make $X$ a timelike vector field. The main difference with respect to the Wick rotation is that it preserves the signature of the metrics. Namely, the rescaled metric $h=R_{(X, \alpha, \beta)}(g)$ is determined by properties 1., 2. (the same used to define the Wick rotation) and

3'. $h(X, X)=\beta g(X, X)$.

The Wick rotation rescaling theory is developed through the following scheme:

- Every maximal globally hyperbolic flat spacetime homeomorphic to $S \times \mathbb{R}$ is proved to be equipped with a $\mathrm{C}^{1,1}$ cosmological time $T$ (provided that $\pi_{1}(S)$ is not abelian).

- A canonical Wick rotation on $M(>1):=T^{-1}((1,+\infty))$ directed along the gradient of $T$ is shown to yield a hyperbolic metric. Moreover this 
hyperbolic structure extends to a (complex) projective structure on the level surface $M(1)=T^{-1}(1)$.

- A canonical rescaling on $M(<1):=T^{-1}((0,1))$ directed along the gradient of $T$, and yielding a de Sitter metric, is pointed out. Such a de Sitter spacetime, denoted by $M^{(1)}$, turns out to be maximal globally hyperbolic, the level surfaces $M(a)$ of $T$ are Cauchy surfaces, and its cosmological time is an explicit function of $T$.

- A canonical rescaling on $M$ directed along the gradient of $T$ yields an antide Sitter structure denoted by $M^{(-1)}$. Level surfaces of $T$ are Cauchy surfaces for $M^{(-1)}$ and its cosmological time is an explicit function of $T$. It is not maximal but it coincides with the past part of its maximal extension (that is denoted by $\mathcal{N}\left(\mathcal{M}^{(-1)}\right)$ ).

In this context the word canonical means that a function $f: M \rightarrow N$ between two flat globally hyperbolic spacetimes is an isometry if and only if it is an isometry for the respective Wick rotated (or rescaled) structures.

The Wick rotation-rescaling theory leads to the following classification theorem.

Theorem N.9.1. Let $S$ be a surface with non-abelian fundamental group. Then the maps

$$
\begin{aligned}
& \mathcal{M S}_{0}(S) \ni M \mapsto M(1) \in P(S) \\
& \mathcal{M S}_{0}(S) \ni M \mapsto M^{(1)} \in \mathcal{M} \mathcal{S}_{1}(S) \\
& \mathcal{M S}_{0}(S) \ni M \mapsto \mathcal{N}\left(M^{(-1)}\right) \in \mathcal{M S}_{-1}(S)
\end{aligned}
$$

are bijective.

Remark. In $\S 7$, Mess relates the classification of $\mathcal{M S}_{-1}(S)$ to the earthquake theory on $S$. In fact, the holonomy of $M \in \mathcal{M S}_{-1}(S)$ is given by a pair of Fuchsian representations $\left(\rho_{L}, \rho_{R}\right)$ of $S$ (this makes sense because of the natural identification of the isometry group of the Klein model of anti-de Sitter space with $P S L(2, \mathbb{R}) \times P S L(2, \mathbb{R}))$. Moreover, the universal covering $\tilde{M}$ of $M \in \mathcal{M S}_{-1}(S)$ is a convex domain in the Klein model of anti-de Sitter space and its extension to the boundary (which is canonically identified with $\mathbb{R} \mathbb{P}^{1} \times \mathbb{R} \mathbb{P}^{1}$ ) is the graph of the unique homeomorphism of $\mathbb{R P}^{1}$ conjugating $\rho_{L}$ and $\rho_{R}$.

Let $\lambda$ be the measured geodesic lamination of $F_{L}=\mathbb{H}^{2} / \rho_{L}$ such that the left earthquake along it sends $F_{L}$ to $F_{R}=\mathbb{H}^{2} / \rho_{R}$. Denote by $F_{+}$the surface obtained by a left earthquake on $F_{L}$ along $\lambda / 2$. Then the level set $T^{-1}(\pi / 2) \subset M$ is obtained by bending (in a suitable sense) $F_{+}$into $M$ along the measured geodesic lamination of $F_{+}$corresponding to $\lambda$.

When $S$ is not closed, the relationship between anti-de Sitter geometry and hyperbolic geometry is more involved. In fact, on the one hand it is well-known that there are measured geodesic laminations on $S$ that do not give rise to a genuine earthquake. On the other hand it is no longer true that the closure of the universal covering of $M \in \mathcal{M S}_{-1}(S)$ is the graph of a homeomorphism (in general it is just a nowhere-timelike simple curve).

For every measured geodesic lamination on a straight convex set, a generalized earthquake is defined as an injective, but in general not surjective, map of the straight convex set on which the lamination is defined into $\mathbb{H}^{2}$. The boundary curves of the universal covering of maximal globally hyperbolic anti-de Sitter spacetimes can be regarded as the trace at infinity of such generalized earthquakes. 
In particular such a curve is the graph of a homeomorphism if and only if the corresponding generalized earthquake is surjective.

Remark. The class of maximal globally hyperbolic anti-de Sitter spacetimes is invariant under the reversal of time-orientation. On the other hand, the sub-class of those corresponding to genuine measured geodesic laminations on the whole $\mathbb{H}^{2}$ is not invariant under that operation. Such a phenomenon is shown by deepening the example suggested by Mess of the hyperbolic thrice-punctured sphere bent along geodesics joining the punctures. Indeed it is somehow related to the fact that in general earthquakes are not surjective.

Remark. Let us consider a closed surface $S$ and $M \in \mathcal{M S}_{k}(S)$. Since the gradient of the cosmological time $T$ is a unitary vector field, the following formula holds

$$
\operatorname{Vol}(a, b)=\int_{a}^{b} A(t) \mathrm{d} t
$$

where $V(a, b)$ is the volume of $T^{-1}(a, b)$ and $A(t)$ is the area of the surface $T^{-1}(t)$. Since the level set $T^{-1}(t)$ is (up to rescaling) the grafting of a hyperbolic surface $F$ along a measured geodesic lamination $g(t) \lambda$ (where $g$ is an explicit positive function of time) the area of $T^{-1}(t)$ is a function of the length of $\lambda$.

In such a way, formulas are given in 25] which explicitly compute $V(a, b)$ in terms of the Mess parameters of $M$. In particular for $k=-1$, the computation allows one to compute the volume of $T^{-1}(0, \pi / 2)$.

Remark. Let $S$ be a closed surface of genus at least 2 . For any hyperbolic structure $F$ on $S$ and any measured geodesic lamination $\lambda$ on $F$, denote by $M^{(k)}(F, \lambda) \in$ $\mathcal{M S}_{k}(S)$ the spacetime corresponding to the pair $(F, \lambda)$. For every $t>0$ let us consider the spacetime $N_{t}=\frac{1}{t^{2}} M^{(k)}(F, t \lambda)$ obtained by rescaling the metric on $M^{(k)}(F, t \lambda)$ by the factor $1 / t^{2}$. By means of the canonical Wick Rotation rescaling theory one can see that (in a suitable sense) we have

$$
N_{t} \rightarrow M^{(0)}(F, \lambda)
$$

as $t \rightarrow 0$.

\section{N.10. Constant mean Curvature foliations}

Hypersurfaces of constant mean curvature. Let $M$ be a spacelike hypersurface with timelike unit normal $T$, in a Lorentz spacetime $V$, with metric $\langle\cdot, \cdot\rangle$ and covariant derivative $D$. The second fundamental form of $M$ is $K(X, Y)=\left\langle T, D_{X} Y\right\rangle$ and the mean curvature is $\operatorname{tr} K=\sum K\left(e_{i}, e_{i}\right)$ where the sum is over an orthonormal frame of $M . M$ is called a constant mean curvature hypersurface if $d \operatorname{tr} K=0$. Let $\operatorname{Area}(M)$ denote the area of $M$ with respect to the induced volume element. If $\operatorname{tr} K=0, M$ maximizes area with respect to compactly supported variations, and hypersurfaces with $\operatorname{tr} K=0$ are therefore called maximal. A hypersurface with constant mean curvature $\operatorname{tr} K=\tau$ on the other hand maximizes the action

$$
L(M)=\operatorname{Area}(M)+\tau \operatorname{Vol}\left(M ; M_{0}\right)
$$

with respect to compactly supported variations. Here $\operatorname{Vol}\left(M, M_{0}\right)$ is the volume in $V$ bounded by $M$ and some fixed, suitably chosen $M_{0}$. 
The mean curvature of a graph $t=f\left(x^{1}, \ldots, x^{n}\right)$ in $n+1$ dimensional Minkowski space is given by

$$
\mathcal{M}[f]=\sum_{i} D_{i}\left(\frac{D_{i} f}{\sqrt{1-|D f|^{2}}}\right)
$$

Thus the equation $\mathcal{M}[f]$ is a quasilinear (non-uniformly) elliptic operator which therefore satisfies the strong maximum principle. The strong maximum principle holds with rather mild regularity assumptions; see [8].

The strong maximum principle allows one to construct barriers for the variational problem with action $L$, and then results from geometric measure theory allow one to prove existence of smooth solutions. This was carried out by Gerhardt [56] in the setting that is relevant here.

Let $V$ be a spatially compact maximal globally hyperbolic flat (MGHF) spacetime. A point in $V$ is on at most one Cauchy surface with mean curvature $\tau$, unless $V$ splits as a metric product. In this case, the spacetime is of translational type, see below, and the only hypersurfaces with constant mean curvature are maximal, $\operatorname{tr} K=0$.

Let $M$ be a CMC Cauchy surface of dimension $n$ in a spatially compact MGHF spacetime $V$. By viewing the development $\mathcal{D}(V)$ of $V$ as a subset of Minkowski space $\mathbb{M}^{n+1}$, the universal covering $\tilde{M}$ of $M$, which is in a natural way a subset of $\mathcal{D}(V)$, can be considered as a CMC hypersurface in Minkowski space $\mathbb{M}^{n+1}$. It follows from the construction that $\tilde{M}$ is complete. CMC hypersurfaces in Minkowski space are convex, with non-positive Ricci curvature. Further, the Gauss map $\phi: \tilde{M} \rightarrow \mathbb{H}^{n}$ is harmonic. This was apparently first noticed by T. K. Milnor 83 in the 2dimensional case.

CMC foliations of flat spacetimes. We consider spatially compact MGHF spacetimes. By the classification due to Scannell 91] in dimension $3+1$ and Barbot [17] for general dimension, these are, up to finite coverings and linear twisted products (see [17]), either products of spatially compact MGHF spacetimes with Cauchy surface of hyperbolic type, with the Euclidean torus, or of translation type. A spacetime is of translation type if it is a quotient of a flat spacetime of topology $\mathbb{R} \times T^{n}$, with the product metric. It follows from the above discussion that unless the spacetime is of translation type, the mean curvature foliation defines a time function $\tau$ on $V$.

Consider spatially compact MGHF spacetimes of dimension $\geq 2+1$ with Cauchy surface of hyperbolic type. Existence of global CMC foliations was proved by Andersson, Moncrief and Tromba 9 in the $2+1$ dimensional case (and any cosmological constant), assuming existence of one CMC hypersurface. A proof of existence of global CMC foliations of $2+1$ dimensional spacetimes was given by Barbot et al. [18 using level sets of the cosmological time function [7] as barriers. A proof of existence of global CMC foliations of spacetimes of general dimension assuming hyperbolic spatial topology was given by Andersson [4]. The general case was considered by Barbot [17.

In case the spacetime has hyperbolic spatial topology, the CMC hypersurfaces are strictly convex, i.e. $K(X, Y) \leq \lambda\langle X, Y\rangle$ for some $\lambda<0$ and Ric $<0$ for the induced metric. In case the spacetime has a torus factor in the sense discussed above, the constant mean curvature hypersurfaces split as a metric product of a Ricci negative factor with a flat torus factor. This follows from the work in [4]. 


\section{Asymptotics.}

The expanding direction. Assume the spacetime $V$ has hyperbolic spatial topology with Cauchy surface $M$. Let $\Gamma$ be the linear part of the holonomy representation of the fundamental group of $V$. The quotient $\mathbb{H}^{n} / \Gamma$ defines a hyperbolic metric $\gamma$ on $M$, with sectional curvature -1 .

Let $g_{\tau}$ be the metric induced on the $\tau$-level set of the CMC foliation of $V$. As $\tau \nearrow 0$, then $\left(M, g_{\tau}\right)$ expands. However, the scale invariant metric $\tau^{2} g_{\tau}$ converges in the Gromov sense to

$$
\lim _{\tau / 0} \frac{\tau^{2}}{n^{2}} g_{\tau} \rightarrow \gamma
$$

More generally, suppose $V$ has a factor with hyperbolic spatial topology $N$ of dimension $m+1$ and a torus factor of dimension $k$. Let $(N, \gamma)$ be the hyperbolic geometry determined as above. In this case, the $n=m+k$ dimensional scale invariant geometry $\left(M, \frac{\tau^{2}}{n^{2}} g_{\tau}\right)$ collapses and converges in the Gromov sense, as $\tau \nearrow$ 0 , to the $m$-dimensional space $(N, \gamma)$.

The collapsing direction. The development $\mathcal{D}(V)$ is a convex subset of Minkowski space. The singularity of $V$ can be identified with $P=B / \sim$ where $B$ is the boundary of $V$ and $\sim$ is an equivalence relation; see [34. It was shown by Benedetti and Guadagnini [26] that the scale invariant geometry on the level sets of the cosmological time function converges in the Gromov sense to that of $P$, which in the $2+1$ dimensional case can be identified with a real tree.

It has been shown by Andersson 6 that for simplicial spacetimes generated by deforming a Lorentz cone spacetime with respect to a finite collection of nonintersecting totally geodesic hypersurfaces, the above picture holds for the CMC foliation, and the conjecture stated by Benedetti and Guadagnini [26] is valid in this case.

The Gauss map. The Gauss map $\phi: \tilde{M} \rightarrow \mathbb{H}^{n}$ is equivariant with respect to the action of the isometry group on $\tilde{M} \subset \mathcal{D}(V)$. Therefore, in the case where the spatial topology is hyperbolic, $\phi$ defines a Gauss map $\phi: M \rightarrow \mathbb{H}^{n} / \Gamma$ where $\Gamma$ is the linear part of the holonomy group of $V . \phi:\left(M, g_{\tau}\right) \rightarrow(M, \gamma)$ is a harmonic diffeomorphism, which is isotopic to the identity map.

The reduced Hamiltonian. Suppose $V$ is a spatially compact MGHF spacetime, not of translational type. The mean curvature of the unique global CMC foliation is a time function and the flow $\tau \mapsto g_{\tau}$ is the solution to the Einstein evolution equation with the CMC gauge. By choosing a suitable time gauge $t=f(\tau)$, one finds that the reduced Hamiltonian is

$$
\mathcal{H}=|\tau|^{n} \operatorname{Area}\left(M, g_{\tau}\right)
$$

This scale invariant quantity is related to the Yamabe invariant (also known as the $\sigma$-invariant, in fact [4, $\S 1.2]$

$$
\inf _{g, K} \frac{n-1}{n} \mathcal{H}^{2 / n} \geq-\sigma(M)
$$

and in case the Cauchy surface is of hyperbolic type, one conjectures

$$
\inf _{g, K} \mathcal{H}=n^{n} \operatorname{Area}(M, \gamma)
$$


where $\gamma$ is a hyperbolic metric on $M$ with sectional curvature -1 . Note that $\operatorname{Area}(M, \gamma)$ is a topological invariant.

By [4, we have

$$
\lim _{\tau / 0} \mathcal{H}(g, K)=n^{n} \operatorname{Area}(M, \gamma)
$$

in case the Cauchy surface is of hyperbolic type.

More generally, when $M$ has a torus factor, the same argument as in 4] gives

$$
\lim _{\tau \nearrow 0} \mathcal{H}(g, K)=0
$$

since in this case, the torus factor collapses when viewed in the rescaled geometry.

\section{REFERENCES}

1. S. Åminneborg, I. Bengtsson, D. R. Brill, S. Holst, and P. Peldán, Black holes and wormholes in $2+1$ dimensions, Classical Quantum Gravity 15 (1998), no. 3, 627-644.

2. S. Åminneborg, I. Bengtsson, and S. Holst, A spinning anti-de Sitter wormhole, Classical Quantum Gravity 16 (1999), no. 2, 363-382.

3. S. Åminneborg, I. Bengtsson, S. Holst, and P. Peldán, Making anti-de Sitter black holes, Classical Quantum Gravity 13 (1996), no. 10, 2707-2714.

4. L. Andersson, Constant mean curvature foliations of flat space-times, Comm. Anal. Geom. 10 (2002), no. 5, 1125-1150.

5. L. Andersson, The global existence problem in general relativity, The Einstein equations and the large scale behavior of gravitational fields (P. T. Chruściel and H. Friedrich, eds.), Birkhäuser, Boston-Basel-Berlin, 2004, pp. 71-120.

6. L. Andersson, Constant mean curvature foliations of simplicial flat spacetimes, arXiv:math.DG/0307338, to appear, Comm. Anal. Geom., 2005.

7. L. Andersson, G. J. Galloway, and R. Howard, The cosmological time function, Classical Quantum Gravity 15 (1998), no. 2, 309-322.

8. _ A strong maximum principle for weak solutions of quasi-linear elliptic equations with applications to Lorentzian and Riemannian geometry, Comm. Pure Appl. Math. 51 (1998), no. $6,581-624$.

9. L. Andersson, V. Moncrief, and A. J. Tromba, On the global evolution problem in $2+1$ gravity, J. Geom. Phys. 23 (1997), no. 3, 191-205.

10. B. N. Apanasov, Nontriviality of Teichmüller space for Kleinian group in space, Riemann surfaces and related topics: Proceedings of the 1978 Stony Brook conference (I. Kra and B. Maskit, eds.), Ann. of Math. Stud., vol. 97, Princeton Univ. Press, Princeton, 1981, pp. 2131 .

11. B. N. Apanasov, The geometry of Nielsen's hull for a Kleinian group in space and quasiconformal mappings, Ann. Global Anal. Geom. 6 (1988), no. 3, 207-230.

12. L. Auslander, On radicals of discrete subgroups of Lie groups, Amer. J. Math. 85 (1963), no. $2,145-150$.

13. M. Bañados, M. Henneaux, C. Teitelboim, and J. Zanelli, Geometry of the $2+1$ black hole, Phys. Rev. D 48 (1993), no. 4, 1506-1525.

14. M. Bañados, C. Teitelboim, and J. Zanelli, Black hole in three-dimensional spacetime, Phys. Rev. Lett. 69 (1992), no. 13, 1849-1851.

15. T. Barbot, Causal properties of AdS-isometry groups, I: Causal actions and limit sets, arXiv:math.GT/0509552, 2005.

16. Causal properties of AdS-isometry groups, II: BTZ multi black-holes, arXiv:math.GT/0510065, 2005.

17. _ Globally hyperbolic flat space-times, J. Geom. Phys. 53 (2005), no. 2, 123-165.

18. T. Barbot, F. Béguin, and A. Zeghib, Feuilletages des espaces temps globalement hyperboliques par des hypersurfaces à courbure moyenne constante, C. R. Acad. Sci. Paris Sér. I Math. 336 (2003), no. 3, 245-250.

19. Constant mean curvature foliations of globally hyperbolic spacetimes locally modelled on $A d S_{3}$, to appear in Geom. Dedicata, this issue, 2005. 
20. T. Barbot and A. Zeghib, Group actions on Lorentz spaces, mathematical aspects: a survey, The Einstein equations and the large scale behavior of gravitational fields (P. T. Chruściel and H. Friedrich, eds.), Birkhäuser, Boston-Basel-Berlin, 2004, pp. 401-439.

21. S. Baseilhac and R. Benedetti, Quantum hyperbolic invariants of 3-manifolds with PSL(2, C)characters, Topology 43 (2004), no. 6, 1373-1423.

22. Classical and quantum dilogarithmic invariants of flat $P S L(2, \mathbb{C})$-bundles over 3manifolds, Geom. Topol. 9 (2005), 493-569.

23. Luantum hyperbolic geometry, arXiv:math/0611504. To appear, Algebr. Geom. Topol., 2006.

24. J. K. Beem, P. E. Ehrlich, and K. L. Easley, Global Lorentzian geometry, 2 ed., Monogr. Textbooks Pure Appl. Math, vol. 202, Marcel Dekker, Inc., New York, 1996.

25. R. Benedetti and F. Bonsante, Canonical Wick rotations in 3-dimensional gravity, arXiv:math.DG/0508485. To appear, Mem. Amer. Math. Soc., 2005.

26. R. Benedetti and E. Guadagnini, Cosmological time in $(2+1)$-gravity, Nuclear Phys. B 613 (2001), 330-352.

27. A. N. Bernal and M. Sánchez, On smooth Cauchy hypersurfaces and Geroch's splitting theorem, Comm. Math. Phys. 243 (2003), no. 3, 461-470.

28. — Smooth globally hyperbolic splittings and temporal functions, arXiv:gr-qc/0404084, 2004.

29. Smoothness of time functions and the metric splitting of globally hyperbolic spacetimes, Comm. Math. Phys. 257 (2005), no. 1, 43-50.

30. F. Bonahon, Shearing hyperbolic surfaces, bending pleated surfaces and Thurston's symplectic form, Ann. Fac. Sci. Toulouse Math. 5 (1996), no. 2, 233-297.

31. K Kleinian groups which are almost Fuchsian, J. Reine Angew. Math. 587 (2005), $1-15$.

32. F. Bonahon and X. Liu, Representations of the quantum Teichmüller space, and invariants of surface diffeomorphisms, arXiv:math.GT/0407086, 2004.

33. F. Bonahon and J. P. Otal, Laminations measurées de plissage des variétés hyperboliques de dimension 3, Ann. of Math. (2) 160 (2004), no. 3, 1013-1055.

34. F. Bonsante, Flat spacetimes with compact hyperbolic Cauchy surfaces, J. Differential Geom. 69 (2005), no. 3, 441-521.

35. - Measured geodesic lamination on straight convex sets and regular domains in Minkowski space, In preparation, 2006.

36. F. Bonsante, K. Krasnov, and J.-M. Schlenker, Multi black holes and earthquakes on Riemann surfaces with boundaries, arXiv:math.GT/0610429, 2006.

37. F. Bonsante and J.-M. Schlenker, AdS manifolds with particles and earthquakes on singular surfaces, arXiv:math.GT/0609116, 2006.

38. D. R. Brill, Multi-black-hole geometries in $(2+1)$-dimensional gravity, Phys. Rev. D 53 (1996), no. 8, 4133-4137.

39. D. R. Brill, Black holes and wormholes in $2+1$ dimensions, Mathematical and quantum aspects of relativity and cosmology (S. Cotsakis and G. W. Gibbons, eds.), Lecture Notes in Phys., vol. 537, Springer-Verlag, New York-Berlin-Heidelberg, 2000, pp. 143-179.

40. K. Bromberg, Hyperbolic cone-manifolds, short geodesics, and Schwarzian derivatives, J. Amer. Math. Soc. 17 (2004), no. 4, 783-826.

41. M. Burger, A. Iozzi, F. Labourie, and A. Wienhard, Maximal representations of surface groups: symplectic Anosov structures, arXiv:math.DG/0506079, 2005.

42. S. Carlip, Quantum gravity in $2+1$ dimensions, Cambridge Univ. Press, Cambridge, 1998.

43. Y. Carrière, Autour de la conjecture de L. Markus sur les variétés affines, Invent. Math. 95 (1989), no. 3, 615-628.

44. Y. Choquet-Bruhat and R. P. Geroch, Global aspects of the Cauchy problem in General Relativity, Comm. Math. Phys. 14 (1969), 329-335.

45. T. A. Drumm, Fundamental polyhedra for Margulis space-times, Topology 31 (1992), no. 4, 677-683.

46. T. A. Drumm and W. M. Goldman, Complete flat Lorentz 3-manifolds with free fundamental group, Israel J. Math. 1 (1990), no. 2, 149-161.

47. D. B. A. Epstein and A. Marden, Convex hulls in hyperbolic space, a theorem of Sullivan, and measured pleated surfaces, Analytical and geometric aspects of hyperbolic space (D. B. A. 
Epstein, ed.), London Math. Soc. Lecture Note Ser., vol. 111, Cambridge Univ. Press, Cambridge, 1987, pp. 113-254.

48. V. V. Fock and L. O. Chekhov, Quantum Teichmüller spaces, Theoret. and Math. Phys. 120 (1999), no. 3, 1245-1259.

49. V. V. Fock and A. B. Goncharov, Dual Teichmüller and lamination spaces, arXiv:math.DG/0510312, 2005.

50. Y. Fourès-Bruhat, Théorème d'existence pour certains systèmes d'équations aux dérivées partielles non linéaires, Acta Math. 88 (1952), 141-225.

51. D. L. Fried and W. M. Goldman, Three-dimensional affine crystallographic groups, Adv. Math. 47 (1983), no. 1, 1-49.

52. D. M. Gallo, Prescribed holonomy for projective structures on compact surfaces, Bull. Amer. Math. Soc. (N.S.) 20 (1989), no. 1, 31-34.

53. D. M. Gallo, W. M. Goldman, and R. M. Porter, Projective structures with monodromy in $\operatorname{PSL}(2, \mathbb{R})$, Unpublished.

54. D. M. Gallo, M. È. Kapovich, and A. Marden, The monodromy groups of Schwarzian equations on closed Riemann surfaces, Ann. of Math. (2) 151 (2000), no. 2, 625-704.

55. D. Garfinkle and S. G. Harris, Ricci fall-off in static and stationary, globally hyperbolic, non-singular spacetimes, Classical Quantum Gravity 14 (1997), no. 1, 139-151.

56. C. Gerhardt, H-surfaces in Lorentzian manifolds, Comm. Math. Phys. 89 (1983), no. 4, 523-553.

57. R. P. Geroch, Domain of dependence, J. Math. Phys. 11 (1970), no. 2, 437-449.

58. W. M. Goldman, Projective structures with Fuchsian holonomy, J. Differential Geom. 25 (1987), no. 3, 297-326.

59. - Topological components of spaces of representations, Invent. Math. 93 (1988), no. 3, $557-607$.

60. W. M. Goldman and G. A. Margulis, Flat Lorentz 3-manifolds and cocompact Fuchsian groups, Crystallographic groups and their generalizations, Contemp. Math., vol. 262, Amer. Math. Soc., Providence, 2000, pp. 135-145.

61. S. G. Harris, Complete codimension-one spacelike immersions, Classical Quantum Gravity 4 (1987), no. 6, 1577-1585.

62. S. G. Harris and R. J. Low, Causal monotonicity, omniscient foliations and the shape of space, Classical Quantum Gravity 18 (2001), no. 1, 27-43.

63. S. W. Hawking and G. F. R. Ellis, The large scale structure of space-time, Cambridge Univ. Press, Cambridge, 1973.

64. D. A. Hejhal, Monodromy groups and linearly polymorphic functions, Acta Math. 135 (1975), $1-55$.

65. A. Ishibashi, T. Koike, M. Siino, and S. Kojima, Compact hyperbolic universe and singularities, Phys. Rev. D 54 (1996), no. 12, 7303-7310.

66. Y. Kamishima and S. P. Tan, Deformation spaces on geometric structures, Aspects of lowdimensional manifolds (Y. Matsumoto and S. Morita, eds.), Adv. Stud. Pure Math., vol. 20, Kinokuniya, Tokyo, 1992, pp. 263-299.

67. R. M. Kashaev, Quantization of Teichmüller spaces and the quantum dilogarithm, Lett. Math. Phys. 43 (1998), no. 2, 105-115.

68. B. Klingler, Complétude des variétés Lorentziennes à courbure constante, Math. Ann. 306 (1996), no. 2, 353-370.

69. S. Kobayashi and K. Nomizu, Foundations of differential geometry, 1 ed., vol. 1, Wiley, New York, 1963.

70. K. Krasnov and J.-M. Schlenker, Minimal surfaces and particles in 3-manifolds, arXiv:math.DG/0511441, 2005.

71. R. S. Kulkarni and U. Pinkall, Uniformization of geometric structures with applications to conformal geometry, Differential geometry, Peñiscola, 1985 (A. M. Naveira, A. Ferrández, and F. Mascaró, eds.), Lecture Notes in Math., vol. 1209, Springer-Verlag, New York-BerlinHeidelberg, 1986, pp. 190-209.

72. R. S. Kulkarni and U. Pinkall, A canonical metric for Möbius structures and its applications, Math. Z. 216 (1994), no. 1, 89-129.

73. F. Labourie, Métriques prescrites sur le bord des variétés hyperboliques de dimension 3, J. Differential Geom. 35 (1992), no. 3, 609-626. 
74. Surfaces convexes dans l'espace hyperbolique et $\mathbb{C} P^{1}$-structures, J. London Math. Soc. (2) 45 (1992), 549-565.

75. L Fuchsian affine actions of surface groups, J. Differential Geom. 59 (2001), no. 1, $15-31$.

76. C. Lecuire, Plissage des variétés hyperboliques de dimension 3, To appear, Invent. Math., 2002.

77. — Mixing invariants of hyperbolic 3-manifolds, C.R.M. preprint núm. 716, 2006.

78. A. Marden, On homotopic mappings of Riemann surfaces, Ann. of Math. (2) 90 (1969), no. 1, $1-8$.

79. E. Martinec, Soluble systems in quantum gravity, Phys. Rev. D 30 (1984), no. 6, 1198-1204.

80. S. Matsumoto, Some remarks on foliated $S^{1}$ bundles, Invent. Math. 90 (1987), no. 2, 343-358.

81. C. T. McMullen, The moduli space of Riemann surfaces is Kähler hyperbolic, Ann. of Math. (2) 151 (2000), no. 1, 327-357.

82. G. Mess, Lorentz spacetimes of constant curvature, MSRI Preprint 90-05808, 1990.

83. T. Klotz Milnor, Harmonic maps and classical surface theory in Minkowski 3-space, Trans. Amer. Math. Soc. 280 (1983), no. 1, 161-185.

84. V. Moncrief, Reduction of the Einstein equations in $2+1$ dimensions to a Hamiltonian system over Teichmüller space, J. Math. Phys. 30 (1989), no. 12, 2907-2914.

85. M. M. Morrill, Non-existence of compact de Sitter manifolds, Ph.D. thesis, University of California, Los Angeles, 1996.

86. R. Penrose, Some unsolved problems in classical general relativity, Seminar on Differential Geometry (S.-T. Yau, ed.), Ann. of Math. Stud., vol. 102, Princeton Univ. Press, Princeton, 1982, pp. 631-668.

87. F. Salein, Variétés anti-de Sitter de dimension 3 possédant un champ de Killing non trivial, C. R. Acad. Sci. Paris Sér. I Math. 324 (1997), no. 5, 525-530.

88. - Variétés anti-de Sitter de dimension 3 exotiques, Ann. Inst. Fourier (Grenoble) 50 (2000), no. 1, 257-284.

89. K. P. Scannell, Flat conformal structures and causality in de Sitter manifolds, Ph.D. thesis, University of California, Los Angeles, 1996

90. _ Flat conformal structures and the classification of de Sitter manifolds, Comm. Anal. Geom. 7 (1999), no. 2, 325-345.

91. 3-manifolds which are spacelike slices of flat spacetimes, Classical Quantum Gravity 18 (2001), no. 9, 1691-1701.

92. K. P. Scannell and M. Wolf, The grafting map of Teichmüller space, J. Amer. Math. Soc. 15 (2002), no. 4, 893-927.

93. J.-M. Schlenker, Hyperbolic manifolds with convex boundary, Invent. Math. 163 (2006), no. 1, 109-169.

94. C. M. Series, Thurston's bending measure conjecture for once punctured torus groups, arXiv:math.GT/0406056, 2004

95. S. P. Tan, Representations of surface groups into $\operatorname{PSL}(2, \mathbb{R})$ and geometric structures, Ph.D. thesis, University of California, Los Angeles, 1988.

96. (1994), no. 1, 649-667.

97. J. W. Wood, Bundles with totally disconnected structure group, Comment. Math. Helv. 46 (1971), 257-273.

98. A. Zeghib, On closed anti-de Sitter spacetimes, Math. Ann. 310 (1998), no. 4, 695-716. 
Department of Mathematics, University of Miami, Coral Gables, FL 33124, USA and Albert Einstein Institute, am Mühlenberg 1, D-144476 Potsdam, Germany

E-mail address: larsa@math.miami.edu

CNRS, UMR 5669, Ecole Normale Supérieure de Lyon, 46 allée d'Italie, 69364 Lyon

E-mail address: Thierry.Barbot@umpa.ens-lyon.fr

Dipartimento di Matematica, Università di Pisa, Largo B. Pontecorvo, 5, I-56127 Pisa

E-mail address: benedett@dm.unipi.it

Dipartimento di Matematica, Universitì di Pisa, Largo B. Pontecorvo, 5, I-56127 Pisa

E-mail address: f.bonsante@sns.it

Department of Mathematics, University of Maryland, College Park, MD 20742

E-mail address: wmg@math.umd.edu

Topologie et Dynamique, Université Paris-Sud, F-91405 Orsay (Cedex)

E-mail address: francois.labourie@math.u-psud.fr

Department of Mathematics and Computer Science, Saint Louis University, St. Louis, MO 63103

E-mail address: scannell@slu.edu

Institut de Mathématiques, Université Paul Sabatier, 31062 Toulouse Cedex 9

E-mail address: schlenker@picard.ups-tlse.fr 